\title{
On a restriction problem of de Leeuw type for Laguerre multipliers
}

\author{
George Gasper円 and Walter Trebels \\ Dedicated to Károly Tandori on the occasion of his 70th birthday
}

(Aug. 10, 1994 version)

\begin{abstract}
In $1965 \mathrm{~K}$. de Leeuw [3] proved among other things in the Fourier transform setting: If a continuous function $m\left(\xi_{1}, \ldots, \xi_{n}\right)$ on $\mathbf{R}^{n}$ generates a bounded transformation on $L^{p}\left(\mathbf{R}^{n}\right), 1 \leq p \leq \infty$, then its trace $\tilde{m}\left(\xi_{1}, \ldots, \xi_{m}\right)=m\left(\xi_{1}, \ldots, \xi_{m}, 0, \ldots, 0\right), m<$ $n$, generates a bounded transformation on $L^{p}\left(\mathbf{R}^{m}\right)$. In this paper, the analogous problem is discussed in the setting of Laguerre expansions of different orders.

Key words. Laguerre polynomials, embeddings of multiplier spaces, projections, transplantation, weighted Lebesgue spaces
\end{abstract}

AMS(MOS) subject classifications. 33C45, 42A45, 42C10

\section{Introduction}

The purpose of this paper is to discuss the question: suppose $\left\{m_{k}\right\}_{k \in \mathbf{N}_{0}}$ generates a bounded transformation with respect to a Laguerre function expansion of order $\alpha$ on some $L^{p}$-space, does it also generate a corresponding bounded map with respect to a Laguerre function expansion of order $\beta$ ? To become more precise let us first introduce some notation. Consider the Lebesgue spaces

$$
\begin{aligned}
& L_{w(\gamma)}^{p}=\left\{f:\|f\|_{L_{v(\gamma)}^{p}}=\left(\int_{0}^{\infty}\left|f(x) e^{-x / 2}\right|^{p} x^{\gamma} d x\right)^{1 / p}<\infty\right\}, \quad 1 \leq p<\infty \\
& L_{w(\gamma)}^{\infty}=\left\{f:\|f\|_{L_{w(\gamma)}^{\infty}}=\operatorname{ess}_{\sup _{x>0}}\left|f(x) e^{-x / 2}\right|<\infty\right\}, \quad p=\infty,
\end{aligned}
$$

where $\gamma>-1$. Let $L_{n}^{\alpha}(x), \alpha>-1, n \in \mathbf{N}_{0}$, denote the classical Laguerre polynomials (see Szegö [15, p. 100]) and set

$$
R_{n}^{\alpha}(x)=L_{n}^{\alpha}(x) / L_{n}^{\alpha}(0), \quad L_{n}^{\alpha}(0)=A_{n}^{\alpha}=\left(\begin{array}{c}
n+\alpha \\
n
\end{array}\right)=\frac{\Gamma(n+\alpha+1)}{\Gamma(n+1) \Gamma(\alpha+1)} .
$$

\footnotetext{
${ }^{1}$ Department of Mathematics, Northwestern University, Evanston, IL 60208, USA. The work of this author was supported in part by the National Science Foundation under grant DMS-9103177.

${ }^{2}$ Fachbereich Mathematik, TH Darmstadt, Schloßgartenstr.7, D-64289 Darmstadt, Germany.
} 
Associate to $f$ its formal Laguerre series

$$
f(x) \sim(\Gamma(\alpha+1))^{-1} \sum_{k=0}^{\infty} \hat{f}_{\alpha}(k) L_{k}^{\alpha}(x),
$$

where the Fourier Laguerre coefficients of $f$ are defined by

$$
\hat{f}_{\alpha}(n)=\int_{0}^{\infty} f(x) R_{n}^{\alpha}(x) x^{\alpha} e^{-x} d x
$$

(if the integrals exist). A sequence $m=\left\{m_{k}\right\}_{k \in \mathbf{N}_{0}}$ is called a (bounded) multiplier from $L_{w(\gamma)}^{p}$ into $L_{w(\delta)}^{q}$, notation $m \in M_{\alpha ; \gamma, \delta}^{p, q}$, if

$$
\left\|\sum_{k=0}^{\infty} m_{k} \hat{f}_{\alpha}(k) L_{k}^{\alpha}\right\|_{L_{w(\delta)}^{q}} \leq C\left\|\sum_{k=0}^{\infty} \hat{f}_{\alpha}(k) L_{k}^{\alpha}\right\|_{L_{w(\gamma)}^{p}}
$$

for all polynomials $f$; the smallest constant $C$ for which this holds is called the

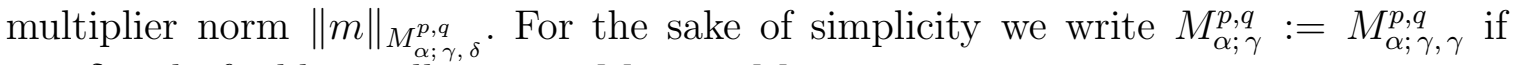
$\gamma=\delta$ and, if additionally $p=q, M_{\alpha ; \gamma}^{p}:=M_{\alpha ; \gamma}^{p, p}$.

We are mainly interested in the question: when is $M_{\alpha ; \alpha}^{p, q}$ continuously embedded in $M_{\beta ; \beta}^{p, q}$ :

$$
M_{\alpha ; \alpha}^{p, q} \subseteq M_{\beta ; \beta}^{p, q}, \quad 1 \leq p \leq q \leq \infty ?
$$

The Plancherel theory immediately yields

$$
l^{\infty}=M_{\alpha ; \alpha}^{2}=M_{\beta ; \beta}^{2}, \quad \alpha, \beta>-1 .
$$

A combination of sufficient multiplier conditions with necessary ones indicates which results are to be expected. To this end, define the fractional difference operator $\Delta^{\delta}$ of order $\delta$ by

$$
\Delta^{\delta} m_{k}=\sum_{j=0}^{\infty} A_{j}^{-\delta-1} m_{k+j}
$$

(whenever the series converges), the classes $w b v_{q, \delta}, 1 \leq q \leq \infty, \delta>0$, of weak bounded variation (see [5]) of bounded sequences which have finite norm $\|m\|_{q, \delta}$, where

$$
\begin{array}{ll}
\|m\|_{q, \delta}:=\sup _{k}\left|m_{k}\right|+\sup _{N \in \mathbf{N}_{0}}\left(\sum_{k=N}^{2 N}\left|(k+1)^{\delta} \Delta^{\delta} m_{k}\right|^{q} \frac{1}{k+1}\right)^{1 / q}, & q<\infty, \\
\|m\|_{\infty, \delta}:=\sup _{k}\left|m_{k}\right|+\sup _{N \in \mathbf{N}_{0}}\left|(k+1)^{\delta} \Delta^{\delta} m_{k}\right| & q=\infty .
\end{array}
$$

Observing the duality (see [14])

$$
M_{\alpha ; \gamma}^{p}=M_{\alpha ; \alpha p^{\prime}-\gamma p^{\prime} / p}^{p^{\prime}}, \quad-1<\gamma<p(\alpha+1)-1, \quad 1<p<\infty,
$$


where $1 / p+1 / p^{\prime}=1$, we may restrict ourselves to the case $1<p<2$. The Corollary $1.2 \mathrm{~b}$ ) in [14] gives the embedding

$$
M_{\alpha ; \alpha}^{p} \subseteq w b v_{p^{\prime}, s}, \quad s=(2 \alpha+2 / 3)(1 / p-1 / 2), \quad \alpha>-1 / 3,
$$

when $(2 \alpha+2)(1 / p-1 / 2)>1 / 2$. Theorem 5 in [5] gives the first embedding in

$$
w b v_{p^{\prime}, s} \subseteq w b v_{2, s} \subseteq M_{\beta ; \beta}^{p},
$$

whereas the last one follows from Corollaries 1.2 and 4.5 in [14] provided $s>\max \{(2 \beta+$ $2)(1 / p-1 / 2), 1\}, \beta>-1$. Hence, choosing $\gamma=\alpha$ in (2), we obtain

Proposition 1.1 Let $1<p<\infty$ and $\alpha$ be such that $(2 \alpha+2 / 3)|1 / p-1 / 2|>1$. Then

$$
M_{\alpha ; \alpha}^{p} \subseteq M_{\beta ; \beta}^{p}, \quad-1<\beta<\alpha-2 / 3 .
$$

In the same way we can derive a result for $M^{p, q}$-multipliers. The necessary condition in [6, Cor. 1.3] can easily be extended in the sense of [6, Cor. 2.5 b)] to

$$
\sup _{k}\left|(k+1)^{\sigma} m_{k}\right|+\sup _{n}\left(\sum_{k=n}^{2 n}\left|(k+1)^{\sigma+s} \Delta^{s} m_{k}\right|^{q^{\prime}} / k\right)^{1 / q^{\prime}} \leq C\|m\|_{M_{\alpha ; \alpha}^{p, q}},
$$

where $\alpha>-1 / 3,1 / q=1 / p-\sigma /(\alpha+1), 1<p<q<2,(\alpha+1)(1 / q-1 / 2)>1 / 4$, and $s=(2 \alpha+2 / 3)(1 / q-1 / 2)>0$. Using this and the sufficient condition for $M_{\beta ; \beta}^{p, q}$-multipliers given in [4, Cor. 1.2], which is proved only for $\beta \geq 0$, we obtain

$$
M_{\alpha ; \alpha}^{p, q} \subseteq M_{\beta ; \beta}^{p, q}, \quad 0 \leq \beta<\alpha-2 / 3,(2 \alpha+2 / 3)(1 / q-1 / 2)>1,1<p<q<2 .
$$

In this context let us mention that the same technique yields for $1<p, q<2$

$$
M_{\alpha ; \alpha}^{p} \subseteq M_{\beta ; \beta}^{q}, \quad(2 \alpha+2 / 3)(1 / p-1 / 2)>\max \{(2 \beta+2)(1 / q-1 / 2), 1\} .
$$

This embedding is in so far interesting as it allows to go from $p, 1<p<2$, to $q \neq p, 1<q<2$, connected with a loss in the size of $\beta$ if $q<p$ or a gain in $\beta$ if $1<p<q<2$; e.g.

$$
M_{10 ; 10}^{4 / 3} \subseteq M_{5 ; 5}^{q}, \quad 1.08 \leq q \leq 2, \quad \text { or } \quad M_{2 ; 2}^{8 / 7} \subseteq M_{4 ; 4}^{q}, \quad 3 / 2 \leq q \leq 2 .
$$

Improvements of (44) can be expected by better necessary conditions and/or better sufficient conditions; but this technique cannot give something like

$$
M_{\alpha ; \alpha}^{p} \subseteq M_{\beta ; \beta}^{q}, \quad(\alpha+1)(1 / p-1 / 2)>(\beta+1)(1 / q-1 / 2), 1<p, q<2,
$$


which is suggested by (4) when choosing "large" $\alpha$ with $p$ near 2 since then the number $4(1 / p-1 / 2) / 3$, which describes the smoothness gap between the necessary conditions and the sufficient conditions in [14, Cor. 1.2], is "negligible".

Concerning the general problem "When does $M_{\alpha ; \gamma_{1}, \delta_{1}}^{p, q} \hookrightarrow M_{\beta ; \gamma_{2}, \delta_{2}}^{p, q}$ hold?", we mention results in Stempak and Trebels [14, Cor. 4.3]: For $1<p<\infty$ there holds

$$
M_{\beta ; \beta p / 2+\delta}^{p}=M_{0 ; \delta}^{p} \quad \text { if }\left\{\begin{aligned}
-1-\beta p / 2<\delta<p-1+\beta p / 2, & -1<\beta<0, \\
-1<\delta<p-1, & 0 \leq \beta,
\end{aligned}\right.
$$

which for $\delta=0$ contains Kanjin's [9] result and for $\delta=p / 4-1 / 2$ Thangavelu's [16]. In particular, there holds for $-1<\beta<\alpha, 1<p<\infty$,

$$
M_{\beta ; \beta}^{p}=M_{\beta ; \beta p / 2+\beta p(1 / p-1 / 2)}^{p}=M_{\alpha ; \alpha p / 2+\beta p(1 / p-1 / 2)}^{p}, \quad(2 \beta+2)|1 / p-1 / 2|<1 .
$$

These results are based on Kanjin's [9] transplantation theorem and its weighted version in [14]. The latter gives further insight into our problem in so far as it implies that the restriction $\beta<\alpha-2 / 3$ in Proposition 1.1 is not sharp.

To this end we first note that the following extension of Corollary 4.4 in [14] holds

$$
w b v_{2, s} \subset M_{\alpha ; \alpha p / 2+\eta(p / 2-1)}^{p}, \quad 0 \leq \eta \leq 1, \quad 1<p \leq 2, \quad s>1 / p .
$$

(For the proof observe that for $\alpha=0$ the parameter $\gamma=\eta(p / 2-1), 0 \leq \eta \leq 1$, is admissible in [14, Theorem 1.1] and then follow the argumentation of [14, Cor. 4.4].) This combined with (3) yields for $s=(2 \alpha+2 / 3)(1 / p-1 / 2)>1 / p$

$$
M_{\alpha ; \alpha}^{p} \subset w b v_{2, s} \subset M_{\alpha ; \alpha p / 2+p / 2-1}^{p}, \quad 1<p \leq 2, \quad \alpha>(p+1) /(6-3 p) .
$$

Thus, by interpolation with change of measure,

$$
M_{\alpha ; \alpha}^{p} \subset M_{\alpha ; \alpha p / 2+\delta}^{p}, \quad p / 2-1 \leq \delta \leq \alpha-\alpha p / 2, \quad \alpha>(p+1) /(6-3 p) .
$$

Since (5) gives

$$
M_{\alpha ; \alpha p / 2+\beta p(1 / p-1 / 2)}^{p}=M_{\beta ; \beta}^{p}
$$

we arrive at

Proposition 1.2 Let $1<p \leq 2$ and $\alpha>(p+1) /(6-3 p)$. Then

$$
M_{\alpha ; \alpha}^{p} \subset M_{\beta ; \beta}^{p}, \quad(2 \beta+2)(1 / p-1 / 2)<1, \quad-1<\beta<\alpha .
$$

The first restriction on $\beta$ is equivalent to $\beta<(2 p-2) /(2-p)$. This combined with the restriction on $\alpha$ gives $\alpha-\beta>(7-5 p) /(6-3 p)$, the latter being decreasing in $p$ and taking the value $2 / 3$ at $p=1$. Hence Proposition 1.2 is an improvement of the previous one for all $1<p<2$ provided $(p+1) /(6-3 p)<\alpha \leq(2 p-2) /(2-p)$. 
For big $\alpha$ 's, Proposition 1.1 is certainly better. If in the transplantation theorem in [14 higher exponents could be allowed in the power weight - which is possible in the Jacobi expansion case as shown by Muckenhoupt 12 - the technique just used would give the embedding when $-1<\beta<\alpha, 1<p<2$, and $\alpha>(p+1) /(6-3 p)$.

Summarizing, it is reasonable to

$$
\text { conjecture } \quad M_{\alpha ; \alpha}^{p, q} \subseteq M_{\beta ; \beta}^{p, q}, \quad-1<\beta<\alpha, \quad 1 \leq p \leq q \leq \infty .
$$

Apart from the above fragmentary results, so far we can only prove the conjecture in the extreme case when $q=\infty$ and $\beta \geq 0$; the latter restriction arises from the fact that we have to make use of the twisted Laguerre convolution (see [7]) which is proved till now only for Laguerre polynomials $L_{n}^{\alpha}(x)$ with $\alpha \geq 0$. Our main result is

Theorem 1.3 If $1 \leq p \leq \infty$, then

$$
M_{\alpha ; \alpha}^{p, \infty} \subset M_{\beta ; \beta}^{p, \infty}, \quad 0 \leq \beta<\alpha .
$$

Remarks. 1) One could speculate that an interpolation argument applied to

$$
M_{\alpha ; \alpha}^{2}=M_{\beta ; \beta}^{2}, \quad M_{\alpha ; \alpha}^{\infty}=M_{\alpha ; \alpha}^{1} \subseteq M_{\beta ; \beta}^{1}=M_{\beta ; \beta}^{\infty}, \quad \beta<\alpha,
$$

could give the open case $M_{\alpha ; \alpha}^{p} \subset M_{\beta ; \beta}^{p}, 1<p<2$. In this respect we mention a result of Zafran [17, p. 1412] for the Fourier transform pointed out to us by A. Seeger:

Denote by $M^{p}(\mathbf{R})$ the set of bounded Fourier multipliers on $L^{p}(\mathbf{R})$ and by $M^{\wedge}(\mathbf{R})$ the set of Fourier transforms of bounded measures on $\mathbf{R}$. Then $M^{p}(\mathbf{R}), 1<p<2$, is not an interpolation space with respect to the pair $\left(M^{\wedge}(\mathbf{R}), L^{\infty}(\mathbf{R})\right)$.

Thus de Leeuw's result mentioned at the beginning cannot be proved by interpolation.

2 ) It is perhaps amazing to note that the $w b v$-classes do not play only an auxiliary role in dealing with the above formulated general problem. In the framework of onedimensional Fourier transforms/series this was shown by Muckenhoupt, Wheeden, and Wo-Sang Young [13]. That this phenomenon also occurs in the framework of Laguerre expansions can be seen from the following two theorems.

Theorem 1.4 If $\alpha>-1, \alpha \neq 0$, then

$$
w b v_{2,1} \subset M_{\alpha ; \alpha+1}^{2}
$$

In the case $-1<\alpha<0$ the multiplier operator is defined only on the subspace $\left\{f \in L_{w(\alpha+1)}^{2}: \hat{f}_{\alpha}(0)=0\right\}$. 
Theorem 1.5 If $\alpha>-1$, then

$$
M_{\alpha ; \alpha+1}^{2} \hookrightarrow w b v_{2,1} .
$$

A combination of these two results leads to

$$
M_{\alpha ; \alpha+1}^{2}=M_{\beta ; \beta+1}^{2}=w b v_{2,1}, \quad \alpha, \beta>-1, \quad \alpha, \beta \neq 0,
$$

and a combination with [14, (19)] gives

$$
M_{\alpha ; \alpha+1}^{2} \subseteq M_{\alpha ; \alpha}^{p}, \quad \alpha \geq 0, \quad(2 \alpha+2) /(\alpha+1)<p \leq 2 .
$$

\section{Proof of Theorem 1.3}

Theorem 1.3 is an immediate consequence of the combination of the following two theorems.

Theorem 2.1 Let $f \in L_{w(\alpha)}^{p}$ with $\alpha>-1$ when $1 \leq p<\infty$ and $\alpha \geq 0$ when $p=\infty$. Then there exists a function $g \in L_{w(\beta)}^{p},-1<\beta<\alpha$, with

$$
g(x) \sim(\Gamma(\beta+1))^{-1} \sum_{k=0}^{\infty} \hat{f}_{\alpha}(k) L_{k}^{\beta}(x), \quad\|g\|_{L_{w(\beta)}^{p}} \leq C\|f\|_{L_{w(\alpha)}^{p}} .
$$

\section{Proof}

First let $1 \leq p<\infty$ and, without loss of generality, let $f$ be a polynomial (these are dense in $L_{w(\alpha)}^{p}$. We recall the projection formula (3.31) in Askey and Fitch [2]

$$
e^{-x} L_{n}^{\beta}(x)=\frac{1}{\Gamma(\alpha-\beta)} \int_{x}^{\infty}(y-x)^{\alpha-\beta-1} e^{-y} L_{n}^{\alpha}(y) d y, \quad-1<\beta<\alpha .
$$

Then the following computations are justified.

$$
\begin{aligned}
\|g\|_{L_{w(\beta)}^{p}} & =C\left(\int_{0}^{\infty}\left|\sum_{k=0}^{\infty} \hat{f}_{\alpha}(k) L_{k}^{\beta}(x) e^{-x / 2}\right|^{p} x^{\beta} d x\right)^{1 / p} \\
& =C\left(\int_{0}^{\infty}\left|\int_{x}^{\infty}(y-x)^{\alpha-\beta-1} e^{-y} \sum_{k=0}^{\infty} \hat{f}_{\alpha}(k) L_{k}^{\alpha}(y) d y\right|^{p} x^{\beta} e^{x p / 2} d x\right)^{1 / p} \\
& \leq C \int_{1}^{\infty}(t-1)^{\alpha-\beta-1}\left(\int_{0}^{\infty}\left|\sum_{k} \hat{f}_{\alpha}(k) L_{k}^{\alpha}(x t) x^{\alpha-\beta+\beta / p} e^{-x(t-1 / 2)}\right|^{p} d x\right)^{1 / p} d t
\end{aligned}
$$


after a substitution and application of the integral Minkowski inequality. Additional substitutions lead to

$$
\begin{aligned}
\|g\|_{L_{w(\beta)}^{p} \leq} \leq & C \int_{0}^{\infty} s^{\alpha-\beta-1}(s+1)^{\beta / p^{\prime}-\alpha-1 / p} \times \\
& \left(\int_{0}^{\infty}\left|\sum_{k} \hat{f}_{\alpha}(k) L_{k}^{\alpha}(y) e^{-y / 2} y^{(\alpha-\beta) / p^{\prime}} e^{-y s / 2(s+1)}\right|^{p} y^{\alpha} d y\right)^{1 / p} d s \\
\leq & C \int_{0}^{\infty} s^{(\alpha-\beta) / p-1}(s+1)^{-(\alpha+1) / p}\left(\int_{0}^{\infty}\left|\sum_{k} \hat{f}_{\alpha}(k) L_{k}^{\alpha}(y) e^{-y / 2}\right|^{p} y^{\alpha} d y\right)^{1 / p} d s
\end{aligned}
$$

where we used the inequality $y^{(\alpha-\beta) / p^{\prime}} e^{-y s / 2(s+1)} \leq C((s+1) / s)^{(\alpha-\beta) / p^{\prime}}$. Since $-1<$ $\beta<\alpha$ it is easily seen that the outer integration only gives a bounded contribution. If $f \in L_{w(\alpha)}^{\infty}$ then $\left|(k+1)^{-1 / 2} \hat{f}_{\alpha}(k)\right| \leq C\|f\|_{L_{w(\alpha)}^{\infty}}$ by [10, Lemma 1] and, therefore, the Abel-Poisson means of an arbitrary $f \in L_{w(\alpha)}^{\infty}$ can be represented by

$$
P_{r} f(x)=(\Gamma(\alpha+1))^{-1} \sum_{k} r^{k} \hat{f}_{\alpha}(k) L_{k}^{\alpha}(x), \quad 0 \leq r<1, \quad x \geq 0,
$$

and, by the convolution theorem in Görlich and Markett [7, p. 169],

$$
\left\|P_{r} f\right\|_{L_{w(\alpha)}^{\infty}} \leq C\|f\|_{L_{w(\alpha)}^{\infty}}, \quad 0 \leq r<1, \quad \alpha \geq 0 .
$$

A slight modification of the argument in the case $1 \leq p<\infty$ shows that

$$
\left\|g_{r}\right\|_{L_{w(\beta)}^{\infty}}:=\left\|(\Gamma(\beta+1))^{-1} \sum_{k} r^{k} \hat{f}_{\alpha}(k) L_{k}^{\beta}\right\|_{L_{w(\beta)}^{\infty}} \leq C\left\|P_{r} f\right\|_{L_{w(\alpha)}^{\infty}} \leq C\|f\|_{L_{w(\alpha)}^{\infty}} .
$$

By the weak* compactness there exists a function $g \in L_{w(\beta)}^{\infty}$ with $\hat{g}_{\beta}(k)=\hat{f}_{\alpha}(k)$ and $\|g\|_{L_{w(\beta)}^{\infty}} \leq \liminf _{k \rightarrow \infty}\left\|g_{r_{k}}\right\|_{L_{w(\beta)}^{\infty}}$ for a suitable sequence $r_{k} \rightarrow 1^{-}$; hence also the assertion in the case $p=\infty$.

Theorem 2.2 For $\alpha \geq 0$ there holds

$$
\begin{aligned}
& M_{\alpha ; \alpha}^{1, p}=M_{\alpha ; \alpha}^{p^{\prime}, \infty}=L_{w(\alpha)}^{p}, \quad 1<p \leq \infty, \\
& M_{\alpha ; \alpha}^{1,1}=M_{\alpha ; \alpha}^{\infty, \infty}=\left\{m=\left\{m_{k}\right\}_{k \in \mathbf{N}_{0}}:\left\|P_{r}(m)\right\|_{L_{w(\alpha)}^{1}}=O(1), r \rightarrow 1^{-}\right\},
\end{aligned}
$$

where $P_{r}(m)(x)=(\Gamma(\alpha+1))^{-1} \sum_{k} r^{k} m_{k} L_{k}^{\alpha}(x)$.

\section{Proof}

The first equalities in $i$ ) and $i i$ ) are the standard duality statements. Let us briefly indicate the second equalities (which are also more or less standard).

If $m=\left\{m_{k}\right\}_{k \in \mathbf{N}_{0}}$ are the Fourier Laguerre coefficients of an $L_{w(\alpha)}^{p}$-function, $1<p \leq$ $\infty$, or in the case $p=1$ of a bounded measure with respect to the weight $e^{-x / 2} x^{\alpha}$, then 
Young's inequality in Görlich and Markett [7] (or a slight extension of it to measures in the case $p=1$ ) shows that $m \in M_{\alpha ; \alpha}^{p^{\prime}, \infty}$.

Conversely, associate formally to a sequence $m=\left\{m_{k}\right\}$ an operator $T_{m}$ by

$$
T_{m} f(x) \sim(\Gamma(\alpha+1))^{-1} \sum_{k=0}^{\infty} m_{k} \hat{f}_{\alpha}(k) L_{k}^{\alpha}(x) .
$$

Then, in essentially the notation of Görlich and Markett [7],

$$
T_{m}\left(P_{r} f\right)(x)=P_{r}(m) * f(x)=\int_{0}^{\infty} T_{x}^{\alpha}\left(P_{r}(m)(y)\right) f(y) e^{-y} y^{\alpha} d y
$$

where $T_{x}^{\alpha}$ is the Laguerre translation operator. If $\|f\|_{L_{w(\alpha)}^{p^{\prime}}}=1$ then

$$
\left\|T_{m}\left(P_{r} f\right)\right\|_{L_{w(\alpha)}^{\infty}} \leq\|m\|_{M_{\alpha ; \alpha}^{p^{\prime}, \infty}}\left\|P_{r} f\right\|_{L_{w(\alpha)}^{p^{\prime}}} \leq C\|m\|_{M_{\alpha ; \alpha}^{p^{\prime} ; \infty}}
$$

and hence, by the converse of Hölder's inequality,

$$
\begin{aligned}
& \sup _{\|f\|_{L_{w(\alpha)}^{p^{\prime}}}=1}\left|\int_{0}^{\infty} T_{x}^{\alpha}\left(P_{r}(m)(y)\right) e^{-y / 2} y^{\alpha / p} f(y) e^{-y / 2} y^{\alpha / p^{\prime}} d y\right| \\
& =\left\|T_{x}^{\alpha}\left(P_{r}(m)\right)\right\|_{L_{w(\alpha)}^{p}} \leq C\|m\|_{M_{\alpha ; \alpha}^{p^{\prime}, \infty}}
\end{aligned}
$$

for $x \geq 0,0 \leq r<1$. In particular, for $x=0$ we obtain

$$
\left\|P_{r}(m)\right\|_{L_{w(\alpha)}^{p}} \leq C\|m\|_{M_{\alpha ; \alpha}^{p^{\prime}, \infty}}, \quad 0 \leq r<1
$$

Now weak* compactness gives the desired converse embedding.

\section{Proof of Theorems 1.4 and 1.5}

The proof relies heavily on the Parseval formula

$$
\frac{1}{\Gamma(\alpha+1)} \sum_{k=0}^{\infty} A_{k}^{\alpha}\left|\hat{f}_{\alpha}(k)\right|^{2}=\int_{0}^{\infty}\left|f(x) e^{-x / 2}\right|^{2} x^{\alpha} d x
$$

and its extension

$$
\sum_{k=0}^{\infty} A_{k}^{\alpha+\lambda}\left|\Delta^{\lambda} \hat{f}_{\alpha}(k)\right|^{2} \approx \int_{0}^{\infty}\left|f(x) e^{-x / 2}\right|^{2} x^{\alpha+\lambda} d x, \quad \lambda \geq 0
$$

which is a consequence of the formula

$$
\Delta^{\lambda} \hat{f}_{\alpha}(k)=C_{\alpha, \lambda} \hat{f}_{\alpha+\lambda}(k)
$$


(see e.g. the proof of Lemma 2.1 in [6]). For the proof of Theorem 1.4 we further need the following discrete analog of the $p=2$ case of a weighted Hardy inequality in Muckenhoupt [11] whose proof can at once be read off from [11] by replacing the integrals there by sums and using the fact that

$$
a \leq 2(a+b)^{1 / 2}\left[(a+b)^{1 / 2}-b^{1 / 2}\right]
$$

when $a, b \geq 0$; also see the extensions in [1], Sec. 4].

Lemma 3.1 Let $\left\{u_{k}\right\}_{k \in \mathbf{N}_{0}},\left\{v_{k}\right\}_{k \in \mathbf{N}_{0}}$ be non-negative sequences (if $v_{k}=0$ we set $\left.v_{k}^{-1}=0\right)$. Then

a)

$$
\begin{aligned}
& \sum_{k=0}^{\infty}\left|\sum_{j=0}^{k} a_{j}\right|^{2} u_{k} \leq C \sup _{N}\left(\sum_{k=N}^{\infty} u_{k} \sum_{k=0}^{N} v_{k}^{-1}\right) \sum_{j=0}^{\infty}\left|a_{j}\right|^{2} v_{j} . \\
& \sum_{k=0}^{\infty}\left|\sum_{j=k}^{\infty} a_{j}\right|^{2} u_{k} \leq C \sup _{N}\left(\sum_{k=0}^{N} u_{k} \sum_{k=N}^{\infty} v_{k}^{-1}\right) \sum_{j=0}^{\infty}\left|a_{j}\right|^{2} v_{j} .
\end{aligned}
$$

Proof of Theorem 1.4. Using (9) and the operator $T_{m}$ defined in (7), we obtain

$$
\int_{0}^{\infty}\left|T_{m} f(x) e^{-x / 2}\right|^{2} x^{\alpha+1} d x \approx \sum_{k=0}^{\infty} A_{k}^{\alpha+1}\left|\Delta\left(m_{k} \hat{f}_{\alpha}(k)\right)\right|^{2} .
$$

Since

$$
\Delta\left(m_{k} \hat{f}_{\alpha}(k)\right)=m_{k} \Delta \hat{f}_{\alpha}(k)+\hat{f}_{\alpha}(k+1) \Delta m_{k}
$$

we first observe that

$$
\sum_{k=0}^{\infty} A_{k}^{\alpha+1}\left|m_{k}\right|^{2}\left|\Delta \hat{f}_{\alpha}(k)\right|^{2} \leq\|m\|_{\infty}^{2} \sum_{k=0}^{\infty} A_{k}^{\alpha+1}\left|\Delta \hat{f}_{\alpha}(k)\right|^{2} \leq C\|m\|_{\infty}^{2}\|f\|_{L_{w(\alpha+1)}^{2}}^{2} .
$$

To dominate the term containing $\Delta m_{k}$ we deduce from (8) that for $\alpha \geq 0$ the Fourier Laguerre coefficients tend to zero as $k \rightarrow \infty$. Hence

$$
\sum_{k=0}^{\infty} A_{k}^{\alpha+1}\left|\hat{f}_{\alpha}(k+1) \Delta m_{k}\right|^{2}=\sum_{k=0}^{\infty} A_{k}^{\alpha+1}\left|\Delta m_{k}\right|^{2}\left|\sum_{j=k+1}^{\infty} \Delta \hat{f}_{\alpha}(j)\right|^{2}=: I .
$$

In order to apply Lemma $3.1 \mathrm{~b}$ ), we choose $u_{k}=A_{k}^{\alpha+1}\left|\Delta m_{k}\right|^{2}$ and $v_{k}=A_{k}^{\alpha+1}$, and observe that when $M \in \mathbf{N}, 2^{M-1} \leq N<2^{M}$, we have that

$$
\begin{aligned}
\left(\sum_{k=0}^{N} u_{k} \sum_{k=N}^{\infty} v_{k}^{-1}\right) & \leq C(N+1)^{-\alpha} \sum_{j=0}^{M} \sum_{k=2^{j}-1}^{2^{j+1}-2}(k+1)\left|\Delta m_{k}\right|^{2} \frac{A_{k}^{\alpha+1}}{k+1} \\
& \leq C(N+1)^{-\alpha} \sum_{j=0}^{M}\left(2^{j}\right)^{\alpha}\|m\|_{2,1}^{2} \leq C\|m\|_{2,1}^{2}
\end{aligned}
$$


uniformly in $N$ if $\alpha>0$. Then Lemma $3.1 \mathrm{~b}$ ) gives

$$
I \leq C\|m\|_{2,1}^{2} \sum_{j=0}^{\infty} A_{j}^{\alpha+1}\left|\Delta \hat{f}_{\alpha}(j)\right|^{2} \leq C\|m\|_{2,1}^{2}\|f\|_{L_{w(\alpha+1)}^{2}}^{2}
$$

by (9). Thus there remains to consider the case $-1<\alpha<0$. For the same choice of $u_{k}$ and $v_{k}$ one easily obtains

$$
\left(\sum_{k=N}^{\infty} u_{k} \sum_{k=0}^{N} v_{k}^{-1}\right) \leq C\|m\|_{2,1}^{2}
$$

Now assume that $\hat{f}(0)=0$. Then we have

$$
\sum_{k=0}^{\infty} A_{k}^{\alpha+1}\left|\hat{f}_{\alpha}(k+1) \Delta m_{k}\right|^{2}=\sum_{k=0}^{\infty} A_{k}^{\alpha+1}\left|\Delta m_{k}\right|^{2}\left|\sum_{j=0}^{k} \Delta \hat{f}_{\alpha}(j)\right|^{2} \leq C\|m\|_{2,1}^{2}\|f\|_{L_{w(\alpha+1)}^{2}}^{2}
$$

where the last estimate follows by Lemma 4.1 a); thus Theorem 1.4 is established.

The proof of Theorem $\mathbf{1 . 5}$ is essentially contained in [6]. As in [6], consider a monotone decreasing $C^{\infty}$-function $\phi(x)$ with

$$
\phi(x)=\left\{\begin{array}{ll}
1 & \text { if } 0 \leq x \leq 2 \\
0 & \text { if } x \geq 4
\end{array}, \quad \phi_{i}(x)=\phi\left(x / 2^{i}\right) .\right.
$$

Then the $\phi_{i}(k)$ are the Fourier Laguerre coefficients of an $L_{w(\alpha+1)}^{2}$-function $\Phi^{(i)}$ with norm $\left\|\Phi^{(i)}\right\|_{L_{w(\alpha+1)}^{2}} \leq C\left(2^{i}\right)^{\alpha / 2}$ and

$$
\begin{aligned}
& \sum_{k=2^{i}}^{2^{i+1}} A_{k}^{\alpha+1}\left|\Delta m_{k}\right|^{2}=\sum_{k=2^{i}}^{2^{i+1}} A_{k}^{\alpha+1}\left|\Delta\left(m_{k} \phi_{i}(k)\right)\right|^{2} \leq \sum_{k=0}^{2^{i+2}} A_{k}^{\alpha+1}\left|\Delta\left(m_{k} \phi_{i}(k)\right)\right|^{2} \\
& \leq C\left\|T_{m} \Phi^{(i)}\right\|_{L_{w(\alpha+1)}^{2}}^{2} \leq C\|m\|_{M_{\alpha ; \alpha+1}^{2}}^{2}\left\|\Phi^{(i)}\right\|_{L_{w(\alpha+1)}^{2}}^{2} \leq C 2^{i \alpha}\|m\|_{M_{\alpha ; \alpha+1}^{2}}^{2}
\end{aligned}
$$

This immediately leads to

$$
\|m\|_{\infty}+\left(\sum_{2^{i}}^{2^{i+1}}\left|(k+1) \Delta m_{k}\right|^{2} \frac{1}{k+1}\right)^{1 / 2} \leq C\|m\|_{M_{\alpha ; \alpha+1}^{2}}
$$

uniformly in $i$, since by $[6,(10)]$ there holds $\|m\|_{\infty} \leq C\|m\|_{M_{\alpha ; \alpha+1}^{2}}$; thus Theorem 1.5 is established.

Remark. 3) (Added on Aug. 10, 1994) The characterization (6) can easily be extended to

$$
M_{\alpha, \alpha+l}^{2}=w b v_{2, l}, \quad \alpha>-1, \quad \alpha \neq 0, \ldots, l-1, l \in \mathbf{N}
$$


In the case $\alpha<l-1$ the multiplier operator is defined only on the subspace $\{f \in$ $\left.L_{w(\alpha+l)}^{2}: \hat{f}_{\alpha}(k)=0,0 \leq k<(l-1-\alpha) / 2\right\}$.

The necessity part carries over immediately (see also [6]). The sufficiency part will be proved by induction. Thus suppose that (12) is true for $l=1, \ldots, n$ and $\alpha$ 's as indicated. Then, as in the case $n=1$, by (9)

$$
\begin{gathered}
\int_{0}^{\infty}\left|T_{m} f(x) e^{-x / 2}\right|^{2} x^{\alpha+n+1} d x \approx \sum_{k=0}^{\infty} A_{k}^{\alpha+n+1}\left|\Delta^{n} \Delta\left(m_{k} \hat{f}_{\alpha}(k)\right)\right|^{2} \\
\leq C \sum_{k=0}^{\infty} A_{k}^{\alpha+n+1}\left|\Delta^{n}\left(m_{k} \Delta \hat{f}_{\alpha}(k)\right)\right|^{2}+C \sum_{k=0}^{\infty} A_{k}^{\alpha+n+1}\left|\Delta^{n}\left(\hat{f}_{\alpha}(k+1) \Delta m_{k}\right)\right|^{2}=: I+I I
\end{gathered}
$$

By the assumption and (10)

$$
\left.I \leq C\|m\|_{w b v_{2, n}}^{2} \sum_{k=0}^{\infty} A_{k}^{\alpha+n+1} \mid \Delta^{n} \hat{f}_{\alpha+1}(k)\right)\left.\right|^{2} \leq C\|m\|_{w b v_{2, n+1}}^{2} \int_{0}^{\infty}\left|f(x) e^{-x / 2}\right|^{2} x^{\alpha+n+1} d x
$$

on account of the embedding properties of the $w b v$-spaces [5]. Analogously $I I$ can be estimated by

$$
I I \leq C\left\|\left\{(k+1) \Delta m_{k}\right\}\right\|_{w b v_{2, n}}^{2} \sum_{k=0}^{\infty} A_{k}^{\alpha+n+1}\left|\Delta^{n}\left(\frac{\hat{f}_{\alpha}(k+1)}{k+1}\right)\right|^{2} .
$$

By the Leibniz formula for differences there holds

$$
\begin{aligned}
\Delta^{n}\left(\frac{\hat{f}_{\alpha}(k+1)}{k+1}\right) & \leq C \sum_{j=0}^{n}\left|\Delta^{j} \hat{f}_{\alpha}(k+1)\right|\left|\Delta^{n-j} \frac{1}{j+k+1}\right| \\
& \leq C \sum_{j=0}^{n}(j+k+1)^{j-n-1}\left|\Delta^{j} \hat{f}_{\alpha}(k+1)\right| .
\end{aligned}
$$

Hence we have to dominate for $j=0, \ldots, n$

$$
I I_{j}:=\sum_{k=0}^{\infty} A_{k}^{\alpha-n-1+2 j}\left|\Delta^{j} \hat{f}_{\alpha}(k+1)\right|^{2}
$$

If $\alpha>n$ then $c_{j}:=-\alpha-2 j+n+1<1$ for all $j=0, \ldots, n, \Delta^{j} \hat{f}_{\alpha}(k+1)=$ $\sum_{i=k+1}^{\infty} \Delta^{j+1} \hat{f}_{\alpha}(i)$, and we can apply [8, Theorem 346] repeatedly to obtain

$$
\begin{aligned}
I I_{j} \leq & C \sum_{k=0}^{\infty} A_{k}^{\alpha-n-1+2 j}\left|(k+1) \Delta^{j+1} \hat{f}_{\alpha}(k+1)\right|^{2} \approx \sum_{k=0}^{\infty} A_{k}^{\alpha-n+2 j+1}\left|\Delta^{j+1} \hat{f}_{\alpha}(k+1)\right|^{2} \\
& \leq \ldots \leq C \sum_{k=0}^{\infty} A_{k}^{\alpha+n+1}\left|\Delta^{n+1} \hat{f}_{\alpha}(k+1)\right|^{2} \leq C \int_{0}^{\infty}\left|f(x) e^{-x / 2}\right|^{2} x^{\alpha+n+1} d x .
\end{aligned}
$$


Since $\left\|\left\{(k+1) \Delta m_{k}\right\}\right\|_{w b v_{2, n}} \leq C\|m\|_{w b v_{2, n+1}}$, this gives the assertion for the weight $x^{n+1}$ in the case $\alpha>n$.

If $\alpha<n, \alpha \neq 0, \ldots, n$, then some $c_{j}>1$. For the application of [8, Theorem 346] one needs $c_{j} \neq 1$; this is guaranteed by the hypothesis $\alpha \neq 0, \ldots, n$ (in the case of an additional weight $x^{n+1}$ ). For the $j$ for which $c_{j}>1$ we have to use the representation

$$
\Delta^{j} \hat{f}_{\alpha}(k+1)=-\sum_{i=0}^{k} \Delta^{j+1} \hat{f}_{\alpha}(i), \quad \text { if } \Delta^{j} \hat{f}_{\alpha}(0)=0,
$$

i.e., the first $(j+1)$ Fourier-Laguerre coefficients have to vanish to ensure this representation. But $0 \leq j \leq j_{0}$, where $j_{0}$ is choosen in such a way that $c_{j_{0}}>1$ and $c_{j_{0}+1}<1$, hence $j_{0}=[(n-\alpha) / 2]$ (with respect to the additional weight $x^{n+1}$ ); here we used the standard notation for $[a], a \in \mathbf{R}$, to be the greatest integer $\leq a$. Hence the condition that the first $[(n-\alpha) / 2]+1$ Fourier-Laguerre coefficients have to vanish is needed if the additional weight is $x^{n+1}$. A repeated application of [8, Theorem 346] with appropriate $c>1$ or $c<1$ now gives the assertion.

\section{References}

[1] K. F. Andersen and H.P. Heinig, Weighted norm inequalities for certain integral operators, SIAM J. Math. Anal., 14 (1983), $834-844$.

[2] R. Askey and J. Fitch, Integral representations for Jacobi polynomials and some applications, J. Math. Anal. Appl., 26 (1969), $411-437$.

[3] K. de Leeuw, On $L^{p}$ multipliers, Ann. of Math., 81 (1965), $364-379$.

[4] G. Gasper, K. Stempak, and W. Trebels, Fractional integration for Laguerre expansions (to appear).

[5] G. Gasper and W. Trebels, A characterization of localized Bessel potential spaces and applications to Jacobi and Hankel multipliers, Studia Math., 65 (1979), $243-278$.

[6] G. Gasper and W. Trebels, On necessary multiplier conditions for Laguerre expansions, Canad. J. Math., 43 (1991), 1228 - 1242.

[7] E. Görlich and C. Markett, A convolution structure for Laguerre series, Indag. Math., 44 (1982), 161 - 171. 
[8] G. H. Hardy, J. E. Littlewood, and G. Polya, Inequalities, Cambridge University Press, Cambridge, 1964.

[9] Y. Kanjin, A transplantation theorem for Laguerre series, Tohoku Math. J., 43 (1991), $537-555$.

[10] C. Markett, Mean Cesàro summability of Laguerre expansions and norm estimates with shifted parameter, Anal. Math., 8 (1982), 19 - 37.

[11] B. Muckenhoupt, Hardy's inequality with weights, Studia Math., 44 (1972), 31 -38 .

[12] B. Muckenhoupt, Transplantation theorems and multiplier theorems for Jacobi series, Mem. Amer. Math. Soc., 64 (1986), no. 356.

[13] B. Muckenhoupt, R.L Wheeden, and Wo-Sang Young, $L^{2}$ multipliers with power weights, Adv. in Math., 49 (1983), $170-216$.

[14] K. Stempak and W. Trebels, On weighted transplantation and multipliers for Laguerre expansions, Math. Ann. (to appear).

[15] G. Szegö, Orthogonal Polynomials, 4th ed., Amer. Math. Soc. Colloq. Publ. 23, Providence, R.I., 1975.

[16] S. Thangavelu, Transplantation, summability and multipliers for multiple Laguerre expansions, Tohoku Math. J., 44 (1992), 279 - 298.

[17] M. Zafran, Interpolation of multiplier spaces, Amer. J. Math., 105 (1983), 1405 -1416 . 